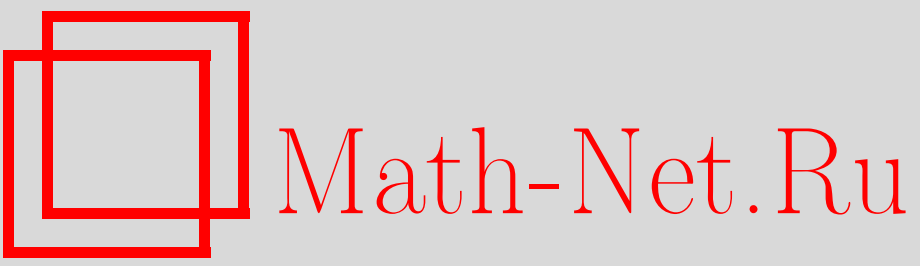

Ю. А. Гладышев, В. В. Калманович, О решении задачи теплопроводности в многослойной среде с фазовыми переходами, Итоги науки и техн. Сер. Соврем. мат. и ее прил. Темат. обз., 2021, том 192, 46-54

DOI: https://doi.org/10.36535/0233-6723-2021-192-46-54

Использование Общероссийского математического портала Math-Net.Ru подразумевает, что вы прочитали и согласны с пользовательским соглашением

http://www.mathnet.ru/rus/agreement

Параметры загрузки:

IP : 54.84 .234 .179

26 апреля 2023 г., 04:57:58 


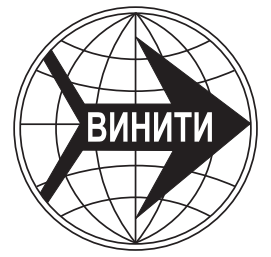

ИТОГИ НАУКИ И ТЕХНИКИ.

Современная математика и ее приложения.

Тематические обзоры.

Том 192 (2021). С. 46-54

DOI: 10.36535/0233-6723-2021-192-46-54

УДК 517.927.2, 517.958, 51-73

\title{
О РЕШЕНИИ ЗАДАЧИ ТЕПЛОПРОВОДНОСТИ В МНОГОСЛОЙНОЙ СРЕДЕ С ФАЗОВЫМИ ПЕРЕХОДАМИ
}

\author{
(c) 2021 г. Ю. А. ГЛАДЫШЕВ, В. В. КАЛМАНОВИЧ
}

\begin{abstract}
АннотАция. В работе решена стационарная задача теплопроводности для случая многослойной среды, состоящей из двух материалов. В рассматриваемой задаче источники тепла находятся в слое, в котором фазовый переход не может произойти, а соседний слой нагревается только за счет теплопроводности, и в нем возможен фазовый переход. Для решения задачи теплопроводности и определения координат точек фазового перехода используется матричный метод совместно с аппаратом обобщенных степеней Берса. Построено поле температур для многослойных сред с различными видами симметрии, когда в каком-либо слое произошел фазовый переход.
\end{abstract}

Ключевые слова: математическая модель, матричный метод, уравнение теплопроводности, многослойная среда, фазовый переход.

\section{ON THE SOLUTION OF THE HEAT-CONDUCTION PROBLEM IN A MULTILAYER MEDIUM WITH PHASE TRANSITIONS}

\author{
(c) 2021 YU. A. GLADYSHEV, V. V. KALMANOVICH
}

\begin{abstract}
In this paper, the stationary problem of heat conduction is solved for the case of a multilayer medium consisting of two materials. In the problem considered, the heat sources are located in a layer in which phase transitions cannot occur, and the neighboring layer is heated only due to thermal conductivity and a phase transition is possible in it. To solve the problem of heat conduction and to determine the coordinates of the points of phase transition, the matrix method is used together with the techniques of generalized Bers degrees. The temperature field is constructed for multilayer media with various types of symmetry, when a phase transition has occurred in some layer.
\end{abstract}

Keywords and phrases: mathematical model, matrix method, heat-conduction equation, multilayer medium, phase transition.

AMS Subject Classification: 34B05, 34B60, 80A20

1. Введение. В современной технике существует много случаев, когда расчет тепловых процессов связан с изучением процессов в многослойных материалах, полученных с определенной целью - создать среду с особыми исключительными характеристиками. Это могут быть различные оболочки, в том числе искривленные: изоляционные покрытия токонесущих тел с осевой симметрией, сферические оболочки, образованные композитными материалами и т. д. Слои этих оболочек могут вследствие нагревания менять свои физические или химические свойства, например, возможны фазовые переходы при определенной температуре. Выяснение возможности и условий таких переходов является важной проблемой. Настоящая работа посвящена исследованию этих вопросов, когда в многослойной среде установилось некоторое конечное стационарное состояние, т.е. будут рассмотрены только стационарные задачи.

Работа выполнена при финансовой поддержке Российского фонда фундаментальных исследований и правительства Калужской области (проекты № 19-03-00271, № 18-41-400001). 
Постановка и решение краевых задач в многослойной среде в случае, когда основные параметры не зависят от температуры, изучены в ряде работ (см., например, [4-7]). Ранее нами было предложено с целью одновременного решения задач для многослойных сред с разной геометрией совместно использовать аппарат обобщенных степеней Берса (см. $[1,8])$ и матричный метод построения решения задач тепломассопереноса (см. $[2,3,9,10]$ ). В данной работе показано, что этот метод может быть использован и для построения решения, когда в каком-то слое происходит фазовый переход.

2. Постановка задачи. Основная система уравнений, определяющая процесс теплопроводности в многослойной среде (номер слоя указан в верхнем индексе в скобках и совпадает с номером его меньшей координаты), имеет вид

$$
D_{2}^{(i)} D_{1}^{(i)} T^{(i)}(x)=-q^{(i)}(x), \quad i=\overline{1, n}
$$

где $D_{1}^{(i)}, D_{2}^{(i)}$ - дифференциальные операторы вида

$$
D_{1}^{(i)}=a_{1}^{(i)}(x) \frac{d}{d x}, \quad D_{2}^{(i)}=a_{2}^{(i)}(x) \frac{d}{d x} .
$$

Положительные функции $a_{1}^{(i)}(x), a_{2}^{(i)}(x)$ определяются геометрическими и физическими параметрами слоев:

$$
a_{1}^{(i)}=\lambda^{(i)} x^{p}, \quad a_{2}^{(i)}=x^{-p},
$$

где $\lambda^{(i)}$ - коэффициент теплопроводности $i$-го слоя. Значение показателя $p$ определяется геометрией среды: значение $p=0$ соответствует процессу теплопроводности в среде со сдвиговой симметрией по оси $x$ (плоские слои), $p=1$-с осевой симметрией (цилиндрические слои) и $p=2-$ с центральной симметрией (сферические слои).

Плотность теплового потока определяется выражением

$$
J^{(i)}(x)=-D_{1}^{(i)} T^{(i)}(x)=-\lambda^{(i)} x^{p} \frac{d}{d x} .
$$

Функция $q^{(i)}(x)$ определяет объемную плотность мощности распределения источников.

На границах слоев приняты условия согласования типа идеального контакта, состоящие в непрерывности температуры и теплового потока

$$
T^{(i)}\left(x_{i+1}\right)=T^{(i+1)}\left(x_{i+1}\right), \quad J^{(i)}\left(x_{i+1}\right)=J^{(i+1)}\left(x_{i+1}\right), \quad i=\overline{1, n-1} .
$$

Рассмотрим процесс теплопроводности в среде, состоящей из двух материалов, когда в одном из материалов есть источники тепла, а в другом нет тепловых источников и возможен фазовый переход при определенной температуре $T_{\mathrm{ph}}$ в некоторой точке $x_{\mathrm{ph}}$. Таким образом, система может моделироваться тремя слоями (рис. 1). Предполагая процесс одномерным и направленным по нормали к оболочке, направим ось $x$ по этой нормали. Пусть $x_{1}, x_{2}, x_{3}, x_{4}$ - координаты границ слоев, причем $x_{1}$ и $x_{4}$-координаты внешних поверхностей. В слое 1 есть источники тепла (например, это проводник, по которому течет электрический ток), а в слоях 2 и 3 источников тепла нет (например, диэлектрик):

$$
q^{(1)}(x)=\text { const }, \quad q^{(2)}(x)=q^{(3)}(x)=0 .
$$

Координата $x_{3}$ - предполагаемая граница фаз при температуре $T^{(3)}=T_{\mathrm{ph}}$ для материала слоев 2 и 3 (искомая величина), значение координаты $x_{2}$ известно. Следовательно, на слоях 2 и 3 коэффициент теплопроводности зависит от температуры:

$$
\lambda(T)= \begin{cases}\lambda^{(2)}, & T<T_{\mathrm{ph}}, \\ \lambda^{(3)}, & T>T_{\mathrm{ph}},\end{cases}
$$

где $\lambda^{(2)}$ и $\lambda^{(3)}$ - постоянные коэффициенты теплопроводности фаз. 


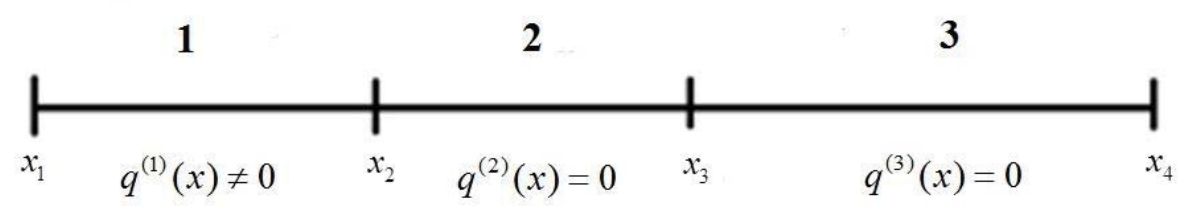

Рис. 1. Модель многослойной среды

Таким образом, для описанной многослойной среды система (1) принимает вид

$$
\begin{aligned}
\frac{d}{d x}\left(\lambda^{(1)} \frac{d T^{(1)}}{d x}\right) & =-q^{(1)}, & & x_{1} \leqslant x \leqslant x_{2}, \\
\frac{d}{d x}\left(\lambda^{(2)} \frac{d T^{(2)}}{d x}\right) & =0, & & x_{2} \leqslant x \leqslant x_{3}, \\
\frac{d}{d x}\left(\lambda^{(3)} \frac{d T^{(3)}}{d x}\right) & =0, & & x_{3} \leqslant x \leqslant x_{4},
\end{aligned}
$$

а условия согласования (3) на границах между слоями -

$$
\begin{gathered}
T^{(1)}\left(x_{2}\right)=T^{(2)}\left(x_{2}\right), \\
T^{(2)}\left(x_{3}\right)=T^{(3)}\left(x_{3}\right)=T_{\mathrm{ph}}, \\
\left.\lambda^{(1)} \frac{d T^{(1)}}{d x}\right|_{x=x_{2}}=\left.\lambda^{(2)} \frac{d T^{(2)}}{d x}\right|_{x=x_{2}}, \\
\left.\lambda^{(2)} \frac{d T^{(2)}}{d x}\right|_{x=x_{3}}=\left.\lambda^{(3)} \frac{d T^{(3)}}{d x}\right|_{x=x_{3}} .
\end{gathered}
$$

На внешних границах $x_{1}$ и $x_{4}$ системы слоев зададим температуры, т.е. поставим первую краевую задачу

$$
T^{(1)}\left(x_{1}\right)=T_{1}, \quad T^{(3)}\left(x_{4}\right)=T_{4}
$$

3. Решение задачи теплопроводности с использованием аппарата обобщенных степеней Берса. Основная трудность решения задачи теплопроводности в многослойной среде состоит в поиске решения, удовлетворяющего внешним граничным условиям (11) и условиям согласования на границах слоев (3), а также поиске координаты возможного фазового перехода $x_{\mathrm{ph}}=x_{3}$, или установления факта того, что фазовый переход не произошел.

Решение задачи будем искать, используя аппарат обобщенных степеней Берса, что позволит в единой аналитической форме охватить случаи различной геометрии слоев. Необходимые сведения об обобщенных степенях приведены ниже:

$$
X_{i}\left(x, x_{i}\right)=\int_{x_{i}}^{x} \frac{d \xi}{a_{1}^{(i)}(\xi)}, \quad \tilde{X}_{i}\left(x, x_{i}\right)=\int_{x_{i}}^{x} \frac{d \xi}{a_{2}^{(i)}(\xi)}, \quad X_{i}^{(2)}\left(x, x_{i}\right)=2 \int_{x_{i}}^{x} \frac{\tilde{X}_{i}\left(\xi, x_{i}\right) d \xi}{a_{1}^{(i)}(\xi)}
$$

В обозначениях обобщенных степеней номер слоя указан в нижнем индексе, а порядок степени в верхнем индексе в скобках, точка $x_{i}$ является нуль-точкой обобщенной степени. 
Например, для плоских слоев (сдвиговая симметрия) формулы (12) принимают вид

$$
\begin{gathered}
X_{i}\left(x, x_{i}\right)=\int_{x_{i}}^{x} \frac{d \xi}{\lambda^{(i)}}=\frac{x-x_{i}}{\lambda^{(i)}}, \quad \tilde{X}_{i}\left(x, x_{i}\right)=\int_{x_{i}}^{x} d \xi=x-x_{i}, \\
X_{i}^{(2)}\left(x, x_{i}\right)=2 \int_{x_{i}}^{x} \frac{\left(\xi-x_{i}\right) d \xi}{\lambda^{(i)}}=\frac{\left(x-x_{i}\right)^{2}}{\lambda^{(i)}},
\end{gathered}
$$

для цилиндрических оболочек (осевая симметрия)

$$
\begin{gathered}
X_{i}\left(x, x_{i}\right)=\int_{x_{i}}^{x} \frac{d \xi}{\lambda^{(i)} \xi}=\frac{1}{\lambda^{(i)}} \ln \frac{x}{x_{i}}, \quad \tilde{X}_{i}\left(x, x_{i}\right)=\int_{x_{i}}^{x} \xi d \xi=\frac{x^{2}-x_{i}^{2}}{2}, \\
X_{i}^{(2)}\left(x, x_{i}\right)=2 \int_{x_{i}}^{x} \frac{\tilde{X}_{i}\left(\xi, x_{i}\right) d \xi}{\lambda^{(i)} \xi}=\frac{1}{\lambda^{(i)}}\left(\frac{x^{2}-x_{i}^{2}}{2}-x_{i}^{2} \ln \frac{x}{x_{i}}\right),
\end{gathered}
$$

а для сферических оболочек (центральная симметрия)

$$
\begin{gathered}
X_{i}\left(x, x_{i}\right)=\int_{x_{i}}^{x} \frac{d \xi}{\lambda^{(i)} \xi^{2}}=\frac{1}{\lambda^{(i)}} \frac{x-x_{i}}{x x_{i}}, \quad \tilde{X}_{i}\left(x, x_{i}\right)=\int_{x_{i}}^{x} \xi^{2} d \xi=\frac{x^{3}-x_{i}^{3}}{3}, \\
X_{i}^{(2)}\left(x, x_{i}\right)=2 \int_{x_{i}}^{x} \frac{\tilde{X}_{i}\left(\xi, x_{i}\right) d \xi}{\lambda^{(i)} \xi^{2}}=\frac{\left(2 x_{i}+x\right)\left(x-x_{i}\right)^{2}}{3 \lambda^{(i)} x} .
\end{gathered}
$$

Обобщенные степени обладают следующими дифференциальными свойствами:

$$
D_{1} X_{i}\left(x, x_{i}\right)=1, \quad D_{2} \tilde{X}_{i}\left(x, x_{i}\right)=1, \quad D_{1} X_{i}^{(2)}\left(x, x_{i}\right)=2 \tilde{X}_{i}\left(x, x_{i}\right) .
$$

В рамках традиционного подхода решение задачи (5)-(11) ищем в виде

$$
\begin{array}{ll}
T^{(1)}(x)=T_{1}+C_{1} X_{1}\left(x, x_{1}\right)-\frac{q^{(1)}}{2} X_{1}^{(2)}\left(x, x_{1}\right), & x_{1} \leqslant x \leqslant x_{2}, \\
T^{(2)}(x)=T_{\mathrm{ph}}+C_{2} X_{2}\left(x, x_{3}\right), & x_{2} \leqslant x \leqslant x_{3}, \\
T^{(3)}(x)=T_{4}+C_{3} X_{3}\left(x, x_{4}\right), & x_{3} \leqslant x \leqslant x_{4},
\end{array}
$$

где $C_{1}, C_{2}, C_{3}$ - произвольные постоянные. Очевидно, что условия (4)-(6), (8), (11) выполнены путем выбора нуль-точек обобщенных степеней - в этом состоит одно из преимуществ использования обобщенных степеней.

Из выражений (13)-(15) согласно (2) найдем плотности тепловых потоков

$$
\begin{aligned}
& J^{(1)}(x)=-C_{1}+q^{(1)} \tilde{X}_{1}\left(x, x_{1}\right), \\
& J^{(2)}(x)=-C_{2}, \\
& J^{(3)}(x)=-C_{3} .
\end{aligned}
$$

Из $(17),(18)$ и $(10)$ получим $C_{3}=C_{2}$. Из (8), (14) и (15) получим

$$
T_{4}+C_{2} X_{3}\left(x_{3}, x_{4}\right)=T_{\mathrm{ph}}
$$

откуда выразим $C_{2}$ :

$$
C_{2}=\frac{T_{\mathrm{ph}}-T_{4}}{X_{3}\left(x_{3}, x_{4}\right)}
$$

Из (16), (17) и условия (9) равенства потоков в точке $x_{2}$ найдем

$$
C_{1}=C_{2}+q^{(1)} \tilde{X}_{1}\left(x_{2}, x_{1}\right)=\frac{T_{\mathrm{ph}}-T_{4}}{X_{3}\left(x_{3}, x_{4}\right)}+q^{(1)} \tilde{X}_{1}\left(x_{2}, x_{1}\right)
$$


Тогда из $(7),(13)$ и $(14)$ с учетом выражений для $C_{1}, C_{2}$ получим

$$
T_{1}+\left(q^{(1)} \tilde{X}_{1}\left(x_{2}, x_{1}\right)+\frac{T_{\mathrm{ph}}-T_{4}}{X_{3}\left(x_{3}, x_{4}\right)}\right) X_{1}\left(x_{2}, x_{1}\right)-\frac{q^{(1)}}{2} X_{1}^{(2)}\left(x_{2}, x_{1}\right)=T_{\mathrm{ph}}+\frac{\left(T_{\mathrm{ph}}-T_{4}\right) X_{2}\left(x_{2}, x_{3}\right)}{X_{3}\left(x_{3}, x_{4}\right)},
$$

откуда

$$
\frac{X_{1}\left(x_{2}, x_{1}\right)-X_{2}\left(x_{2}, x_{3}\right)}{X_{3}\left(x_{3}, x_{4}\right)}=\frac{T_{\mathrm{ph}}-T_{1}+\left(q^{(1)} / 2\right) X_{1}^{(2)}\left(x_{2}, x_{1}\right)-q^{(1)} \tilde{X}_{1}\left(x_{2}, x_{1}\right) X_{1}\left(x_{2}, x_{1}\right)}{T_{\mathrm{ph}}-T_{4}} .
$$

Введем обозначение

$$
s=\frac{T_{1}-T_{\mathrm{ph}}-\left(q^{(1)} / 2\right) X_{1}^{(2)}\left(x_{2}, x_{1}\right)+q^{(1)} \tilde{X}_{1}\left(x_{2}, x_{1}\right) X_{1}\left(x_{2}, x_{1}\right)}{T_{\mathrm{ph}}-T_{4}} .
$$

В результате из уравнения

$$
s X_{3}\left(x_{4}, x_{3}\right)-X_{2}\left(x_{3}, x_{2}\right)=X_{1}\left(x_{2}, x_{1}\right)
$$

определяем неизвестную координату точки фазового перехода $x_{3}$.

Например, в случае плоских слоев получим

$$
x_{3}=\frac{\lambda^{(1)} \lambda^{(2)} x_{4}\left(T_{1}-T_{\mathrm{ph}}+\frac{q^{(1)}\left(x_{2}-x_{1}\right)^{2}}{2 \lambda^{(1)}}\right)+\lambda^{(3)}\left(T_{\mathrm{ph}}-T_{4}\right)\left(\lambda^{(1)} x_{2}-\lambda^{(2)} x_{2}+\lambda^{(2)} x_{1}\right)}{\lambda^{(1)} \lambda^{(2)}\left(T_{1}-T_{\mathrm{ph}}+\frac{q^{(1)}\left(x_{2}-x_{1}\right)^{2}}{2 \lambda^{(1)}}\right)+\lambda^{(1)} \lambda^{(3)}\left(T_{\mathrm{ph}}-T_{4}\right)} .
$$

4. Матричный метод построения решения. Эффективным аналитическим методом построения решения системы (1)-(3) с краевыми условиями первого, второго или третьего типов может быть матричный метод, описанный ранее в ряде статей (см. $[2,3,9])$. Чтобы охватить единым подходом случай искривленных оболочек, совместно с матричным методом используется аппарат обобщенных степеней Берса.

Опишем кратко матричный метод. Рассмотрим систему уравнений (1). На первом сегменте $\left[x_{1}, x_{2}\right]$ поставим задачу Коши, т.е. зададим в начальной точке $x_{1}$ слоя температуру и поток:

$$
\left.T(x)\right|_{x_{1}}=T^{(1)}\left(x_{1}\right),\left.\quad J(x)\right|_{x_{1}}=J^{(1)}\left(x_{1}\right) .
$$

Решение задачи Коши для сегмента $\left[x_{1}, x_{2}\right]$ запишем с помощью аппарата обобщенных степеней Берса:

$$
\begin{aligned}
T^{(1)}(x) & =T^{(1)}\left(x_{1}\right)-J^{(1)}\left(x_{1}\right) X_{1}\left(x, x_{1}\right)-\frac{q^{(1)}}{2} X_{1}^{(2)}\left(x, x_{1}\right), \\
J^{(1)}(x) & =J^{(1)}\left(x_{1}\right)+q^{(1)} \tilde{X}_{1}\left(x, x_{1}\right) .
\end{aligned}
$$

Тогда в конечной точке $x_{2}$ получим

$$
\begin{aligned}
& T^{(1)}\left(x_{2}\right)=T^{(1)}\left(x_{1}\right)-J^{(1)}\left(x_{1}\right) X_{1}\left(x_{2}, x_{1}\right)-\frac{q^{(1)}}{2} X_{1}^{(2)}\left(x_{2}, x_{1}\right), \\
& J^{(1)}\left(x_{2}\right)=J^{(1)}\left(x_{1}\right)+q^{(1)} \tilde{X}_{1}\left(x_{2}, x_{1}\right),
\end{aligned}
$$

т.е. значения температуры и потока в точке $x_{2}$ можно считать заданными. Таким образом, по условиям согласования (3)

$$
T^{(1)}\left(x_{2}\right)=T^{(2)}\left(x_{2}\right), \quad J^{(1)}\left(x_{2}\right)=J^{(2)}\left(x_{2}\right),
$$

на сегменте $\left[x_{2}, x_{3}\right]$ поставлена задача Коши, решение которой есть

$$
\begin{aligned}
T^{(2)}(x) & =T^{(2)}\left(x_{2}\right)-J^{(2)}\left(x_{2}\right) X_{2}\left(x, x_{2}\right)-\frac{q^{(2)}}{2} X_{2}^{(2)}\left(x, x_{2}\right), \\
J^{(2)}(x) & =J^{(2)}\left(x_{2}\right)+q^{(2)} \tilde{X}_{2}\left(x, x_{2}\right) .
\end{aligned}
$$


Следовательно, можно найти значения температуры и потока в точке $x_{3}$, после чего поставить задачу Коши для следующего сегмента. Продолжая аналогичные действия, получим решение для всей многослойной среды.

Введем следующие обозначения для сегмента $\left[x_{i}, x_{i+1}\right]$ :

$$
\begin{aligned}
& V^{(i)}(x)=\left(\begin{array}{c}
T^{(i)}(x) \\
J^{(i)}(x)
\end{array}\right), \quad K^{(i)}\left(x, x_{i}\right)=\left(\begin{array}{cc}
1 & -X_{i}\left(x, x_{i}\right) \\
0 & 1
\end{array}\right), \\
& V^{(i)}\left(x_{i}\right)=\left(\begin{array}{c}
T^{(i)}\left(x_{i}\right) \\
J^{(i)}\left(x_{i}\right)
\end{array}\right), \quad W^{(i)}\left(x, x_{i}\right)=\left(\begin{array}{c}
w^{(i)}(x)-w^{(i)}\left(x_{i}\right) \\
D_{1}^{(i)} w^{(i)}(x)
\end{array}\right),
\end{aligned}
$$

где $w^{(i)}(x)$ - произвольное частное решение уравнения (1) для $i$-го слоя, например,

$$
W^{(i)}\left(x, x_{i}\right)=\left(\begin{array}{c}
-\frac{1}{2} q^{(i)} X_{i}^{(2)}\left(x, x_{i}\right) \\
q^{(i)} \tilde{X}\left(x, x_{i}\right)
\end{array}\right) .
$$

Тогда решение задачи Коши для $i$-го слоя можно записать в матричной форме:

$$
V^{(i)}(x)=K^{(i)}\left(x, x_{i}\right) V^{(i)}\left(x_{i}\right)+W^{(i)}\left(x, x_{i}\right) .
$$

Условия согласования (3) в матричной форме имеют вид

$$
V^{(i)}\left(x_{i+1}\right)=V^{(i+1)}\left(x_{i+1}\right) .
$$

Тогда для первого слоя имеем

$$
V^{(1)}(x)=K^{(1)}\left(x, x_{1}\right) V^{(1)}\left(x_{1}\right)+W^{(1)}\left(x, x_{1}\right), \quad x_{1} \leqslant x \leqslant x_{2} .
$$

Учитывая условия идеального контакта $(20)$ в точке $x_{2}$, для второго слоя получим

$$
\begin{aligned}
V^{(2)}(x)=K^{(2)}\left(x, x_{2}\right) V^{(2)}\left(x_{2}\right)+W^{(2)}\left(x, x_{2}\right)= & \\
& =K^{(2)}\left(x, x_{2}\right)\left[K^{(1)}\left(x_{2}, x_{1}\right) V^{(1)}\left(x_{1}\right)+W^{(1)}\left(x_{2}, x_{1}\right)\right]+W^{(2)}\left(x, x_{2}\right), \quad x_{2} \leqslant x \leqslant x_{3} .
\end{aligned}
$$

Выполняя аналогичные подстановки последовательно в граничных точках, получим общий результат:

$$
\begin{aligned}
V^{(i)}(x)=K^{(i)}\left(x, x_{i}\right) & \ldots K^{(1)}\left(x_{2}, x_{1}\right) V^{(1)}\left(x_{1}\right)+ \\
& +K^{(i)}\left(x, x_{i}\right) \ldots K^{(2)}\left(x_{3}, x_{2}\right) W^{(1)}\left(x_{2}, x_{1}\right)+\ldots+W^{(i)}\left(x, x_{i}\right), \quad x_{i} \leqslant x \leqslant x_{i+1} .
\end{aligned}
$$

Умножение матриц $K^{(i)}\left(x, x_{i}\right)$ не представляет трудностей, так как элементы $X_{i}\left(x, x_{i}\right)$ просто складываются, т.е. произведение матриц $K$ для слоев с $j$-го по $i$-й имеет вид

$$
K^{(i, \ldots, j)}\left(x, x_{j}\right)=\left(\begin{array}{cc}
1 & -\left(X_{i}\left(x, x_{i}\right)+\sum_{l=j}^{i-1} X_{l}\left(x_{l+1}, x_{l}\right)\right. \\
0 & 1
\end{array}\right), \quad 1 \leqslant j<i .
$$

Физический смысл суммы обобщенных степеней в матрице (21) - это сумма термических сопротивлений слоев при их последовательном соединении.

В конечной точке $x_{n+1}$ системы слоев получим

$$
V^{(n)}\left(x_{n+1}\right)=K^{(n, \ldots, 1)}\left(x_{n+1}, x_{1}\right) V^{(1)}\left(x_{1}\right)+K^{(n-1, \ldots, 2)}\left(x_{n}, x_{2}\right) W^{(1)}\left(x_{2}, x_{1}\right)+\ldots+W^{(n)}\left(x_{n+1}, x_{n}\right) \text {. }
$$

Таким образом, соотношение (22), связывая внешние граничные точки многослойной системы, позволяет по любой известной паре из величин $T^{(1)}\left(x_{1}\right), J^{(1)}\left(x_{1}\right), T^{(n)}\left(x_{n+1}\right), J^{(n)}\left(x_{n+1}\right)$ определить однозначно другую неизвестную пару, т.е. позволяет получать решения для первой, второй и третьей краевых задач, в общем случае сводя решение к системе двух линейных уравнений с двумя неизвестными при любом конечном числе слоев.

Вернемся к поставленной задаче (4)-(11) и проблеме поиска точки возможного фазового перехода. Решив краевую задачу матричным методом, получим решение, выраженное через неизвестный параметр $x_{3}$. Значение координаты фазового перехода $x_{\mathrm{ph}}=x_{3}$ найдем из уравнения $T\left(x_{3}\right)=T_{\mathrm{ph}}$. Если решение уравнения не удовлетворяет неравенству $x_{2} \leqslant x_{3} \leqslant x_{4}$, это означает, 

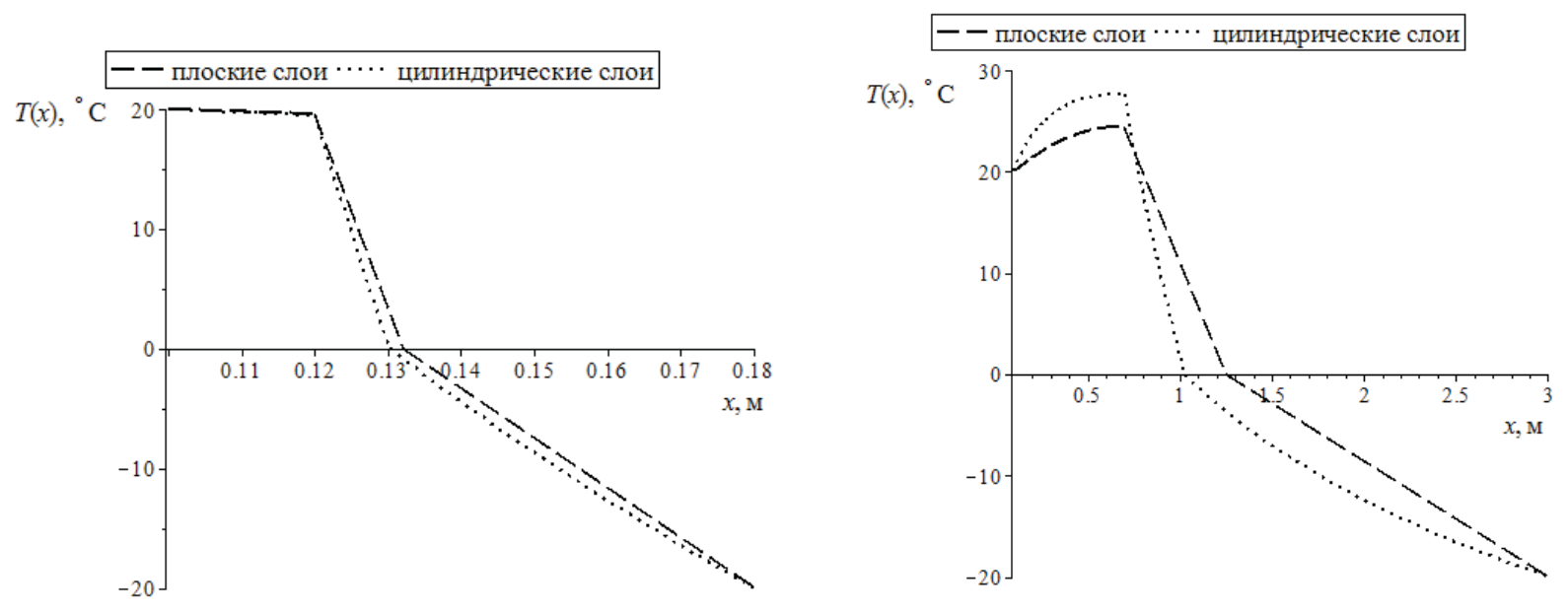

Рис. 2. Решение первой краевой задачи для плоской и цилиндрической многослойных оболочек с фазовым переходом. Координаты границ слоев $x_{1}=0,1 \mathrm{M}, x_{2}=0,12 \mathrm{M}, x_{4}=0,18 \mathrm{м}$
Рис. 3. Решение первой краевой задачи для плоской и цилиндрической многослойных оболочек с фазовым переходом. Координаты границ слоев $-x_{1}=0,1 \mathrm{~m}, x_{2}=0,7 \mathrm{~m}, x_{4}=3 \mathrm{м}$

что фазовый переход не происходит, и точка $x_{3}$ полагается равной $x_{2}$ или $x_{4}$, в зависимости от того, какая фаза будет в слое без источников. Данный результат совпадает с (19).

5. Результаты расчетов и их обсуждение. Рассмотрим результаты моделирования первой краевой задачи (1)-(11) для плоских и цилиндрических оболочек.

Первоначально нами была рассмотрена система «сталь-вода-лед». Для расчетов коэффициент теплопроводности стали принимался равным $\lambda^{(1)}=45,4 \mathrm{BT} /(\mathrm{m} \cdot \mathrm{K})$, воды $-\lambda^{(3)} 2=$ $0,6 \mathrm{Bт} /(\mathrm{м} \cdot \mathrm{K})$, льда $-\lambda^{(2)}=2,3 \mathrm{BT} /(\mathrm{m} \cdot \mathrm{K})$. Границы слоев $-x_{1}=0,1 \mathrm{~m}, x_{2}=0,12 \mathrm{~m}, x_{4}=0,18 \mathrm{м}$. Мощность источников $-q^{(1)}=1200 \mathrm{BT} / \mathrm{m}^{3}$. Заданные температуры $T_{1}=20^{\circ} \mathrm{C}, T_{4}=-20^{\circ} \mathrm{C}$, $T_{\mathrm{ph}}=0^{\circ} \mathrm{C}$. На рис. 2 показаны распределения температуры, полученные при решении задачи матричным методом для плоских слоев и искривленных с осевой симметрией. График распределения температуры представляет непрерывную монотонно убывающую функцию, что говорит об одинаковом направлении теплового потока во всех слоях. На границах слоев выражены изломы, что свидетельствует о разрывах производной в этих точках. Найденная координата точки фазового перехода для плоской системы $x_{\mathrm{ph}}=0,132 \mathrm{м}$, а для цилиндрической оболочки $x_{\mathrm{ph}}=0,130$ м.

При заданных параметрах вид графиков на слое с источниками для системы плоских слоев, а также на всех слоях для цилиндрической оболочки близок к прямым линиям. Однако увеличение толщины слоев ведет к хорошо заметному искривлению графика распределения температуры для этих случаев. На рис. 3 изображены распределения температур при координатах границ слоев $x_{1}=0,1 \mathrm{~m}, x_{2}=0,7 \mathrm{~m}, x_{4}=3$ м и неизменных значениях остальных параметров. На графиках выражен максимум температуры, что говорит о различных направлениях теплового потока в многослойной среде в этом случае. Найденная координата точки фазового перехода для плоской системы $x_{\mathrm{ph}}=1,255 \mathrm{м}$, а для цилиндрической оболочки $x_{\mathrm{ph}}=1,029 \mathrm{м}$.

Поскольку соотношение (22) дает возможность применять матричный метод к различным краевым задачам, то для примера нами также было проведено моделирование распределения температуры в цилиндрической системе слоев «титан- вода - лед» с осью симметрии $x_{1}=0$ при краевых условиях $J^{(1)}\left(x_{1}\right)=0$ - плотность теплового потока на оси симметрии цилиндра, $T^{(3)}\left(x_{4}\right)=T_{4}$ - температура на поверхности цилиндрической системы слоев. На рис. 4 показано распределение температуры, полученное для этого случая при следующих значениях параметров: $\lambda^{(1)}=19,8 \mathrm{BT} /(\mathrm{M} \cdot \mathrm{K})$ (титан), $\lambda^{(3)} 2=0,6 \mathrm{BT} /(\mathrm{м} \cdot \mathrm{K})\left(\right.$ вода), $\lambda^{(2)}=2,3 \mathrm{Bт} /(\mathrm{м} \cdot \mathrm{K})\left(\right.$ лед), $x_{1}=0 \mathrm{м}$, 


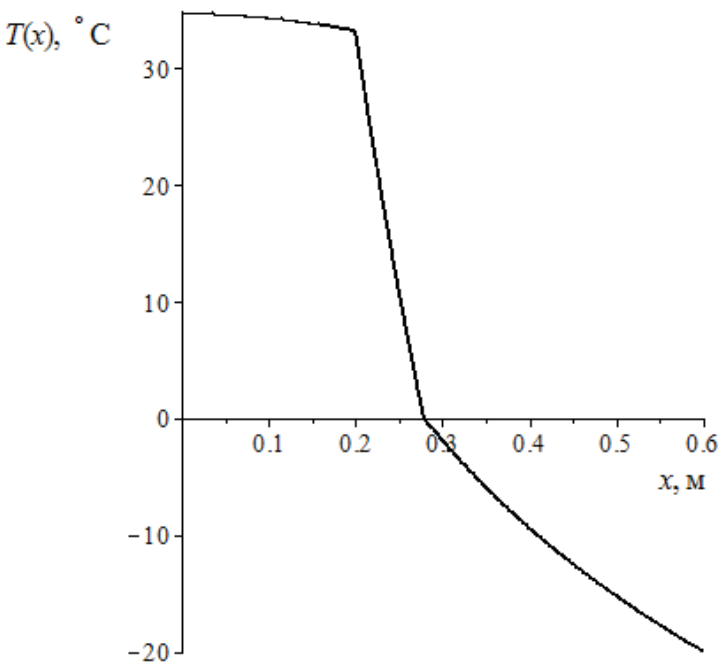

Рис. 4. Распределение температуры в многослойном цилиндре с фазовым переходом.

Границы слоев $x_{1}=0 \mathrm{M}, x_{2}=0,2 \mathrm{M}, x_{4}=0,6 \mathrm{м}$

$x_{2}=0,2 \mathrm{м}, x_{4}=0,6 \mathrm{м}, q^{(1)}=3000 \mathrm{BT} / \mathrm{m}^{3}, J_{1}=0 \mathrm{BT} / \mathrm{m}^{2}, T_{4}=-20^{\circ} \mathrm{C}, T_{\mathrm{ph}}=0^{\circ} \mathrm{C}$. График распределения температуры представляет собой непрерывную функцию с разрывной производной в граничных точках слоев. Функция температуры монотонно убывает, что отражает одинаковое направление потока во всех слоях. Найденная координата точки фазового перехода $x_{\mathrm{ph}}=0,279 \mathrm{~m}$.

6. Заключение. Рассмотренный в работе матричный метод совместно с аппаратом обобщенных степеней Берса позволяет аналитически получить решение задачи теплопроводности в многослойной среде при наличии фазовых переходов. В работе подробно рассмотрено аналитическое решение первой краевой задачи традиционным методом с использованием обобщенных степеней Берса и матричным методом для случая многослойной среды, состоящей из двух материалов, когда источники тепла находятся в слое, в котором фазовый переход не может произойти, а соседний слой нагревается только за счет теплопроводности, и в нем возможен фазовый переход. Расчеты проведены для многослойной среды со сдвиговой и осевой симметрией. Показано, что возможно решение предлагаемым методом и краевых задач второго и третьего типа. Таким образом, этот метод может быть довольно эффективным для решения различных практических задач, например, когда важно определить условия нежелательного фазового перехода при проектировании и эксплуатации различных технических устройств.

\section{СПИСОК ЛИТЕРАТУРЫ}

1. Гладышев Ю. А. Метод обобщенных степеней Берса и его приложение в математической физике. Калуга: КГУ им. К. Э. Циолковского, 2011.

2. Гладышев Ю. А., Калманович В. В., Серегина Е. В., Степович М. А. О возможности совместного применения матричного метода и аппарата обобщенных степеней Берса для математического моделирования процесса теплопереноса в объектах, обладающих цилиндрической симметрией// Вопросы атомн. науки и техн. Сер. Ядерно-реакторные константы. - 2018. - 3. - С. 158-167.

3. Калманович B. B., Степович M. А. О совместном применении матричного метода и аппарата обобщенных степеней Берса для математического моделирования процессов тепломассопереноса в полупроводниковых материалах электронной техники// в кн.: Проблемы разработки перспективных микро- и наноэлектронных систем. - М.: ИППМ РАН, 2018. - Т. III. - С. 194-201.

4. Карслоу Г., Егер Д. Теплопроводность твердых тел. - М.: Наука, 1964.

5. Карташов Э. М. Аналитические методы в теории теплопроводности твердых тел. - М.: Высшая школа, 2001. 
6. Кудинов В. А., Карташов Э. М., Калашников В. В. Аналитические решения задач тепломассопереноса и термоупругости для многослойных конструкций. - М.: Высшая школа, 2005.

7. Лыков А. В. Теория теплопроводности. - М.: Высшая школа, 1967.

8. Bers L., Gelbart A. On a class of functions defined by partial differential equations// Trans. Am. Math. Soc. - 1944. - 56. - P. 67-93.

9. Gladyshev Yu. A., Kalmanovich V. V., Stepovich M. A. On the possibility of applying the Bers apparatus to modeling the processes of heat and mass transfer caused by electrons in a planar multilayer medium// J. Surface Invest. X-Ray, Synch. Neutr. Techniques - 2017. - 11, № 5. - P. 1096-1100.

10. Kalmanovich V. V., Seregina E. V., Stepovich M. A. Mass transfer problem with the combined matrix and generalized powers of the Bers method// J. Phys. Conf. Ser. — 2019. — 1163. — 012012.

Гладышев Юрий Александрович

Калужский государственный университет им. К. Э. Циолковского

E-mail: v572264@yandex.ru

Калманович Вероника Валерьевна

Калужский государственный университет им. К. Э. Циолковского

E-mail: v572264@yandex.ru 